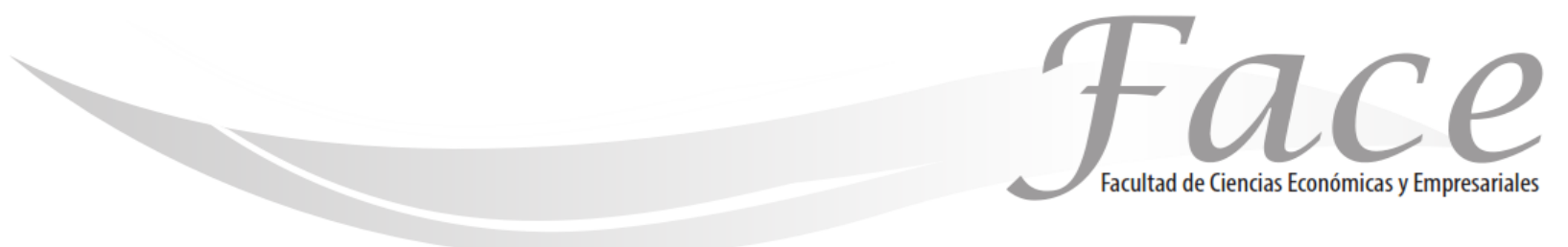

ISSN Impreso: 1794-9920

ISSN Electrónico: 2500-9338

Volumen 21-N¹

Año 2021

Págs. $77-93$

(c)

\title{
EL RÉGIMEN ESPECIAL SOCIETARIO EN COLOMBIA
}

\author{
Ernesto Sandoval Ortega * \\ Enlace ORCID : https://orcid.org/0000-0001-9864-8339 \\ Natalia Margarita Gómez Bolívar ** \\ Enlace ORCID : https://orcid.org/0000-0003-1161-9805
}

Fecha de Recepción: Enero 15 de 2021

Fecha de Aprobación: Mayo 27 de 2021

\section{Resumen:}

Se realiza un estudio teórico y un análisis práctico sobre un tipo de sociedad que en Colombia se crea por una 0 varias personas naturales o jurídicas, con el fin de realizar actividades en beneficio de sus asociados, terceras personas o la comunidad en general, se habla de las "entidades sin ánimo de lucro" (ESAL), el documento también hace énfasis en las donaciones, los incentivos fiscales y el régimen tributario especial $(\mathrm{RTE})$, términos inherentes con este tipo de sociedades con régimen tributario especial.

Se expone de forma clara, cómo ha sido su evolución y la trascendencia que han tenido en el país; el documento muestra como las ESAL resultan ser una excelente opción de desarrollo y un apoyo para las políticas públicas del gobierno, luego de exponer diversos criterios de interés general relacionados con tributos y exoneraciones, se hace precisión sobre este tipo de entidades en el distrito capital de Bogotá, en donde se evidencian en un número considerable, y donde se analizan mediante un instrumento de medición estadística, su estado, cantidad y tipos de empresas bajo esta modalidad. Finalmente se expone un ejemplo de ESAL, y se muestra su participación dentro la sociedad incluso en sectores como el académico mediante alianzas estratégicas.

Palabras clave: ESAL, incentivos, donaciones, régimen tributario especial

\footnotetext{
* Candidato a Doctor, Master Internacional en Auditoria y Gestión Empresarial, Universidad Europea Miguel de Cervantes, España - Valladolid - Castilla y León. Magister en Dirección Estratégica Universidad Internacional Iberoamericana Puerto Rico, Especialista en Auditoria de Sistemas, Especialista en Revisoría Fiscal y Auditoria forense, Contador Público e Ingeniero de Sistemas. Contacto: ernestosandovalortega@gmail.com

${ }^{* *}$ Negocios Internacionales. Estudiante de Facultad de Negocios Internacionales de la Universidad Santo Tomás Seccional Tunja. Investigadora. Practicante de la Fundación Carulla aeioTU en Bogotá Distrito Capital, practicante de Planeación Financiera el año 2019 II. Contacto: natalia.gomez@usantoto.edu.co
} 


\title{
THE SPECIAL SOCIETY REGIME IN COLOMBIA
}

\begin{abstract}
:
A theoretical study and a practical analysis are carried out on a type of society that in Colombia is created by one or more natural or legal persons, in order to carry out activities for the benefit of its associates, third parties or the community in general. of "non-profit entities" (ESAL), the document also emphasizes donations, tax incentives and the special tax regime (RTE), terms inherent with this type of companies with a special tax regime.

It is clearly exposed how its evolution has been and the importance it has had in the country; The document shows how the ESALs turn out to be an excellent development option and a support for the government's public policies, after exposing various criteria of general interest related to taxes and exemptions, it specifies about this type of entities in the capital district of Bogotá, where they are evidenced in a considerable number, and where their status, number and types of companies under this modality are analyzed by means of a statistical measurement instrument. Finally, an example of ESAL is exposed, and its participation within society is shown, even in sectors such as academia through strategic alliances.
\end{abstract}

Keywords: ESAL, incentives, donations, special tax regime

\section{O REGIME ESPECIAL DE SOCIEDADE NA COLÔMBIA}

\section{Resumo:}

Realiza-se um estudo teórico e uma análise prática sobre um tipo de sociedade que na Colômbia é criada por uma ou mais pessoas físicas ou jurídicas, para realizar atividades em benefício de seus associados, terceiros ou da comunidade em geral. das "entidades sem fins lucrativos" (ESAL), o documento destaca ainda as doações, incentivos fiscais e o regime especial de tributação (RTE), termos inerentes a este tipo de empresas com regime especial de tributação.

Mostra-se claramente como tem sido sua evolução e a importância que teve no país; 0 documento mostra como as ESALs se revelaram uma excelente opção de desenvolvimento e um suporte para as políticas públicas do governo, após expor diversos critérios de interesse geral relacionados a impostos e isenções, especifica sobre este tipo de entidades no distrito capital de Bogotá, onde se evidenciam em número considerável, e onde se analisa a sua situação, número e tipo de empresas nesta modalidade por meio de instrumento de medida estatística. Por fim, expõe-se um exemplo da ESAL e mostra-se sua participação na sociedade, inclusive em setores como o acadêmico por meio de alianças estratégicas.

Palavras-chave: ESAL, incentivos, doações, regime tributário especial 


\section{INTRODUCCIÓN:}

En las últimas décadas han emergido con mucha fuerza en el panorama nacional e internacional miles de organizaciones civiles sin ánimo de lucro, e independientes de los gobiernos nacionales, que están teniendo un gran protagonismo social, político y económico. Este fenómeno ha sido denominado por algunos como sociedad civil global (Salamon, 1999) (Kaldor, 2003) y obedece a una especie de revolución asociacional global (Ruiz de Olabuenaga, 2005) o a una verdadera explosión de la sociedad civil (Rosenau, 1997).

Las "entidades del tercer sector" o "entidades sin ánimo de lucro", cuentan con un sistema especial de tributos en Colombia, en los últimos años el gobierno nacional Colombiano ha mostrado un especial interés implementando normativas que aseguran el control al fraude y otros delitos que pueden estar inmersos en la constitución y funcionamiento de este tipo de empresa, intervienen diversos organismos de control como la Unidad Especial Administrativa, DIAN, la cual emite una completa regulación al respecto y que se expone en el presente documento.

Los beneficios adjudicados a este tipo de entidades, pueden ser una de las razones por las que se requiere de un control constante, al mismo tiempo, el Estado colombiano también es consciente de que debe impulsar su creación y sostenimiento a través de ayudas operativas y económicas, además, este tipo de entidades, directa e indirectamente apoyan las políticas gubernamentales impulsando el bienestar social y el mejoramiento de la calidad de vida de comunidades vulnerables y con problemáticas en ámbitos educativos, nutricionales, núcleos familiares, de vivienda, medio ambiente, ciencia y tecnología, y todos los relacionados con ambientes sociales inadecuados sobre todo para la niñez.

El presente documento plantea como objetivo general, la ilustración de los términos propios que se relacionan con las Entidades Sin Ánimo de Lucro -ESAL, y posteriormente presenta una reflexión tomando particularmente un estudio sobre una entidad no lucrativas en el Distrito Capital de Bogotá, este análisis se soporta en las cifras suministradas por el MINTIC (Ministerio de Tecnologías de la Información y las Comunicaciones de Colombia). La ESAL tomada como caso de estuio expone su origen y evolución, y los fines que persigue haciendo país y ayudando a una población que requiere de cuidados especiales. Luego de analizar la situación de este tipo de organizaciones, se pretende dar respuesta a la pregunta: ¿Las ESAL en Colombia aportan al bienestar de poblaciones necesitadas, y el gobierno Nacional apoya su constitución y crecimiento?

\section{Aspectos metodológicos}

Teniendo en cuenta los objetivos planteados, el diseño de la presente investigación es exploratorio ya que el tema propuesto y sus resultados abren el camino para posteriores estratégias investigativas que amplien de forma descriptiva y amplia la problemática aquí expuesta (Batthyány \& Cabrera, 2011).

El Diccionario de la Investigación Científica, de Tamayo y Tamayo (Tamayo y Tamayo, 2004), define al diseño de investigación como «la estructura a seguir en una investigación ejerciendo el control de la misma a fin de encontrar resultados confiables y su relación con los interrogantes surgidos de la hipótesis». $Y$ de acuerdo con Kerlinger el diseño de la investigación es el plan para obtener respuestas a las preguntas de investigación. «Un diseño de investigación expresa la estructura del problema y el plan de investigación usado para obtener evidencia empírica sobre las relaciones del problema» (Kerlinger, 1997).

Los estudios exploratorios sirven para preparar el terreno y por lo común anteceden a investigaciones con alcances descriptivos, correlacionales 0 explicativos (Hernández Sampieri, Fernández Collado, \& Baptista Lucio, 2010) . 
El estudio teórico del artículo lleva a consecución de conceptos y aspectos prácticos relacionados con las ESAL en Colombia, se consultan las fuentes de mayor confiabilidad y pertinencia y se organiza de forma sistemáticamente estructurada para su comprensión y análisis, la población colombiana interesada en la constitución, vigilancia y administración de este tipo de empresa estará satisfecha por los resultados mostrados. busca contribuir al entendimiento de aquellos microprocesos a través de los cuales se desarrolla y mantiene vigente una capacidad organizacional (Dávila, 2012) (Arboleda, 2013).

\section{Reflexión}

\section{Entidades sin Ánimo de Lucro}

Este sector no lucrativo o social, también ha sido reconocido como el "tercer sector" de la economía, pues corresponde a aquel campo residual económico que no corresponde a la economía pública (o conocido también como "primer sector" o "sector institucional"), ni a la economía privada (o también referido como "segundo sector", o "sector comercial" o "capitalista tradicional"). Este tercer sector, por tratarse de una categorización residual, ha servido como término genérico para englobar los muy variados tipos de entidades y organizaciones que, en principio, parecieran no encajar en ninguno de los otros dos sectores (González Robles, 2019). Así, en otras palabras, por simplicidad y practicidad, el término se utiliza recurrentemente para referirse indistintamente a entidades que podrían pertenecer a uno u otro sistema -anglosajón o europeo continental- sin necesidad de revisar detalladamente las características propias 0 detenerse a confirmar dicha calidad con base en ninguna teoría. En Colombia (como constante en muchos escenarios propios de nuestro régimen normativo) no ha existido una clara determinación de la teoría fundante, deviniendo así en dos consecuencias palpables. Por un lado, en la teoría, en una consecuente confusión normativa y conceptual y; por otro lado, en la práctica, en complicaciones de implementación (Niño Mejia, 2017).

Las entidades sin ánimo de lucro (ESAL): "Son personas jurídicas, que pueden ejercer derechos y contraer obligaciones. No pretenden el reparto de los excedentes o utilidades que se generen en desarrollo de su objetivo social, sino que buscan engrandecer su propio patrimonio, para el cumplimiento de sus metas y objetivos que, por lo general, son de beneficio social, bien sea encaminado hacia un grupo determinado de personas o hacia la comunidad en general" (Vargas, 1992).

En otras palabras, una ESAL es aquella organización cuya actividad principal tiene una finalidad social, a partir de una mejora en algún aspecto para un determinado grupo de la sociedad. No distribuye sus ganancias entre sus miembros ni entre sus fundadores, es decir que todas las ganancias 0 donaciones son destinadas al cumplimiento de la función de la organización. (Asociación Nacional de Empresarios de Colombia [ANDI], 2019, p. 2)

La unidad especial administrativa, Dirección de Impuestos y Aduanas Nacionales (DIAN) en Colombia, define la fundación como el ente jurídico surgido de la voluntad de una persona o del querer unitario de varias acerca de su constitución, organización, fines y medios para alcanzarlos. Esa voluntad original se torna irrevocable en sus aspectos esenciales una vez se ha obtenido el reconocimiento como persona jurídica por parte del Estado (Villar, 2001). El substrato de la fundación es la afectación de unos fondos preexistentes a la realización efectiva de un fin de beneficiencia pública, de utilidad común o de interés social (fines educativos, científicos, tecnológicos, culturales, deportivos o recreativos). La irrevocabilidad del querer original no obsta para que el fundador en el acto de constitución se reserve atributos que le permitan interpretar el alcance de su propia voluntad o que lo invistan de categoría orgánica en la administración de la fundación, pero siempre con subordinación al poder constituyente de la voluntad contenida en el acto fundacional y sin que ello implique la existencia de miembros de la institución a cualquier título (DIAN, 2018).

Una organización sin ánimo de lucro (OSAL), también se conoce como organización sin fines de lucro (OSFL), organización no lucrativa (ONL) o entidad sin ánimo de lucro (ESAL) ${ }^{1}$, es una entidad cuyo fin no es la persecución de un beneficio económico sino que principalmente persigue una finalidad social, altruista, humanitaria, artística 0 comunitaria. Este tipo de instituciones por lo general se

${ }^{1}$ En Colombia son llamadas ESAL Entidades sin ánimo de lucro. 
financian gracias a ayudas y donaciones derivada de personas físicas, empresas, instituciones y organizaciones de todo tipo, y en algunos casos (aunque no en todos) también se reciben ayudas estatales puntuales o regulares (en forma de subsidios, usufructo de fincas, exoneraciones fiscales 0 aduaneras, etc.) (iCluster, s.f.)

Estas ESAL son personas jurídicas que se constituyen por la voluntad de asociación o creación de una o más personas (narurales 0 jurídicas) para realizar actividades en beneficio de asociados, terceras personas o comunidad en general por lo tanto no persiguen el reparto de utilidades entre sus miembros (Cámara de Comercio de Bogotá [CCB], s.f.).

Las Entidades sin Ánimo de Lucro, surgen como desarrollo del derecho fundamental de asociación contenido en los artículos 38 y 39 de la Constitución Política, en donde el Estado garantiza la libertad de las personas para reunirse y desarrollar actividades comunes desprovistas del ánimo de lucro. Para ello, el ordenamiento jurídico colombiano exige que dichas entidades nazcan a la vida jurídica como el resultado de la expresión de voluntad para asociarse y la creación de reglas de participación democrática de quienes se asocian. Son personas jurídicas diferentes de las personas que las conforman, (asociados) que pueden ejercer derechos, contraer obligaciones, y estar representadas legal, judicial y extrajudicialmente en virtud del desarrollo y ejecución de las actividades propias de su objeto (Gaitán, 2014).

Una Entidad Sin Ánimo de Lucro se caracteriza por:

- Ser una organización legamente constituida, de carácter civil que se rige por el derecho privado.

- Contar con un patrimonio que está destinado en su totalidad a cumplir con su finalidad.

- Ser una expresión del derecho de asociación y de iniciativa privada.

- Invertir los excedentes que obtenga de forma exclusiva en la realización de su objeto, de forma tal que los mismos, a diferencia de una sociedad comercial, no se reparten o distribuyen entre sus miembros, fundadores o terceros.

- Tener una finalidad que atiende al interés general o beneficia a un número importante de personas, de forma tal que las mismas contribuyen a la realización de los fines del Estado, al adelantar actividades que el Estado no alcanza a desarrollar.
- Promover valores y actitudes de justicia social, equidad, democracia, género, participación y solidaridad en las actividades que desarrolla (Confederación Colombiana de ONG [CCONG], 2016).

\section{MARCO TEÓRICO}

\section{Las Donaciones en Colombia}

Las donaciones con fines caritativos son muy antiguas, y ellas han pasado a ser parte importante de las instituciones sin fines de lucro, que tienen por objetivo la prestación de algún fin benéfico (Sorj, 2007). Las sociedades han ido creando mecanismos para alentarlas a través de ventajas tributarias y estatus legal favorable; sin embargo desde un punto de vista no puramente económico, se atribuye que muchas donaciones tienen como motivaciones de parte de los donantes la satisfacción emotiva y social de altruismo, así como el prestigio que esto pudiera acarrear (Parada, 2001).

El artículo 1443 del Código Civil colombiano, indica: "...La donación entre vivos es un acto por el cual una persona transfiere, gratuita e irrevocablemente, una parte de sus bienes a otra persona que la acepta". De la anterior definición, se interpreta que la transferencia se podrá predicar no solo de la propiedad jurídica de un bien como el derecho real de una cosa corporal, sino también, del derecho a usarlo o explotarlo económicamente sin que lo posea, o lo que también se conoce como la transferencia de la mera o nuda propiedad, según lo indica el artículo 669 del Código Civil (DIAN, 2018).

La donación se entiende como un negocio jurídico en virtud del cual una persona (donante) dispone de una cosa de su patrimonio a favor de otra (donatario) a título gratuito (Martínez Ravé, 2003). Se requiere para su perfección no sólo el animus donandi en el donante, es decir, la liberalidad, sino también la aceptación del donatario de acuerdo con los artículos 618 a 656 deñ Derecho Civil. Es decir, que es un contrato por el cual una persona (donante) trasfiere la propiedad de una bien a otra (donatario) (Juridica., 2021), que la acepta, sin contrapartida y con intención liberal. Es un negocio jurídico por el que una persona, llamada donante, proporciona, a costa de su patrimonio, una cosa o bien a otra persona, llamada donatario, que la acepta. Además de la intención de liberalidad del donante (animus donandi), es preciso que el donatario consienta, pues nadie 
puede influir en el patrimonio de otro si éste no lo permite. No son donación los actos que otorgan una ventaja sin compensación, pero que no ocasionan una pérdida patrimonial apreciable, como sucede en el préstamo sin interés 0 en el comodato (Donación, 2020) (Reyes Villamizar, 2006).

De acuerdo con el artículo 257 del Estatuto Tributario colombiano donde habla sobre los descuentos por donaciones a ESAL pertenecientes al régimen especial, y las donaciones efectuadas a entidades sin ánimo de lucro que hayan sido calificadas en el régimen especial del impuesto sobre la renta y complementarios y a las entidades no contribuyentes de que tratan los artículos $\underline{22}$ y $\underline{23}$ del Estatuto Tributario, no serán deducibles del impuesto sobre la renta y complementarios, pero darán lugar a un descuento del impuesto sobre la renta y complementarios, equivalente al $25 \%$ del valor donado en el año o período gravable. El Gobierno nacional reglamentará los requisitos para que proceda este descuento (Estatuto Tributario, 1989).

La donación es el acto por el que una persona, con ánimo de liberalidad, se empobrece en una fracción de su patrimonio, en provecho de otra persona que se enriquece con ella. Puede definirse a la donación como el acto por el que una persona, con ánimo de liberalidad, se empobrece en una fracción de su patrimonio, en provecho de otra persona que se enriquece con ella (Wolters Kluwer, s.f.).

La norma legal que define las donaciones se encuentra en el articulo 1386 del Código Civil, que señala: "La donación entre vivos es un acto por el cual una persona transfiere gratuita e irrevocablemente una parte de sus bienes a otra persona, que la acepta". La donación es un contrato y, como tal, participan dos partes: el donante, quien realiza una transferencia gratuita e irrevocable de parte de su patrimonio; y el donatario, quien se beneficia recibiendo dichos bienes (Auditoría, Contabilidad \& Finanzas, 2010).

Por tanto, la donación representa el acto de otorgar, generalmente por razones caritativas, algo a otra persona, organización o institución. El capital por su parte representa aquel dinero y los bienes que sustentan o son la base de una persona, empresa u organización. De ese modo, al juntar ambos conceptos, hallamos lo que es la donación de capital, que significa dar fondos 0 bienes materiales que una empresa o particular puede realizar, incluso bajo contrato, dependiendo la legislación que regule al país (Donación de capital, 2019).
Incentivos Fiscales por parte del Estado

A manera de recuento académico, y sin ningún interés en profundizar el debate relativo a la conceptualización estricta de los incentivos tributarios, podríamos decir que existen cuatro categorías diferentes: (1) reducciones directas a las tarifas de tributación; (2) incentivos tributarios propiamente dichos (también reconocidos como minoraciones estructurales); (3) zonas especiales con tratamiento tributario privilegiado; y (4) incentivos al empleo (Niño, 2007).

Este viene siendo un beneficio que se le otorga a alguien para alentarlo a hacer algo específico; utilizar los impuestos como incentivos es una manera en que las empresas e individuos pueden reducir sus impuestos a cambio de acciones deseables específicas o inversiones por su parte (Jaramillo Díaz, Vargas Ballén, \& Durán Acosta, Entidades sin Ánimo de Lucro Régimen Tributario Especial, 1999). El objetivo es incentivar a aquellas empresas e individuos a actuar en forma socialmente responsable y/o beneficiosa para la comunidad. Probablemente la forma más común de incetivo para los contribuyentes estadounidenses son las deducciones por contribuciones benéficas: cuando calcule sus impuestos, usted puede deducir la cifra que donó a organizaciones de caridad exentas de impuestos, usted puede deducir la cifra que donó a organizaciones de caridad exentas de impuestos de su renta imponible. La deducción existe para convencer a las personas de que contribuyan con las organizaciones benéficas (Caja de Herramientas Comunitarias [CHC], s.f.).

Si la donación se ve como un estímulo en forma de bonificaciones en el pago de ciertas obligaciones tributarias que se concede a los sujetos pasivos de dichos tributos, es con el fin de promover la realización de determinadas actividades consideradas de interés por parte del sector público (Incentivo fiscal, s.f.).

Estas disposiciones legales que disminuyen la carga tributaria de un determinado tributo por ciertas conductas o actuaciones de los sujetos gravados, se distinguen por la exigencia de actuaciones o conductas en mérito a las cuáles se establece por la ley la minoración del tributo. Su establecimiento y la delimitación de su alcance están sujetos al principio de reserva de ley (Jaramillo Díaz, 2010). El sentido técnico de este término coincide con su sentido literal. Se trata de disposiciones legales que disminuyen la carga tributaria de un determinado tributo por ciertas conductas 0 actuaciones de los sujetos gravados. En tal medida, forman parte de la categoría más amplia de 
beneficios tributarios, pero el rasgo que los distingue de la especie genérica es esa exigencia de actuaciones o conductas en mérito a las cuáles se establece por la ley la minoración del tributo (Wolters Kluwer, s.f.).

Por un lado, los incentivos tributarios son fáciles de definir. Son disposiciones especiales que establecen exclusiones, créditos, tasas preferentes 0 aplazamientos de obligaciones tributarias (Flórez Gómez, 2018). Los incentivos tributarios pueden adoptar muchas formas: exoneraciones temporales de impuestos, deducciones aplicables a determinados tipos de gastos o reducciones de los aranceles de importación o los derechos de aduana. Por otro lado, puede ser difícil distinguir entre las disposiciones que se consideran parte del régimen general tributario y las que constituyen un tratamiento especial (Caicedo Silva, 2020). Esta distinción será más importante a medida que se limite la capacidad de los países de adoptar incentivos tributarios específicos. Los incentivos tributarios también pueden definirse en función de su capacidad de reducir la carga tributaria efectiva para un proyecto concreto (Naciones Unidas, 2018).

El incentivo fiscal constituye un estímulo por parte del Estado, que se manifiesta como reducción en el pago de determinado tributo. Puede ser aplicado en forma de un porcentaje o monto fijo sobre el total de impuesto a pagar (Belalcázar Erazo \& Ria, 2011). 0 puede ser un crédito fiscal, para ser deducible a futuros pagos. Muchos se plantean la interrogante, ¿los incentivos fiscales son un gasto o una inversión? Lo típico del caso es que, son más los fiscalistas que consideran que los incentivos son un gasto tributario y no una inversión para el Estado. Se sostiene que no debe aumentarse continuamente tales exenciones, que estos recursos deben ser utilizados vía presupuesto nacional, canalizados en actividades productivas; se consideran que tales incentivos son una inversión, que en última instancia promueven el desarrollo económico. Además, los defensores del incentivo fiscal, argumental que el gobierno no es mejor inversor que el sector privado (Fortún, s.f.).

Se definen también como "medidas fiscales utilizadas para atraer inversión de capital local o extranjera a ciertas actividades económicas o áreas particulares en un país" (traducción propia de (Bolnick, 2004). Stotsk y Zee, adoptan un concepto similar, aclarando que "no toda provisión fiscal aplicada a todo proyecto de inversión constituye un incentivo fiscal ya que se excluyen los incentivos fiscales que se aplican de forma general a todas las inversiones" (traducción propia de (Bolnick, 2004). (Asesores y Consultores Internacionales, 2017)

\section{Incentivo Tributario para Empresas de Economía Naranja}

Las rentas provenientes del desarrollo de industrias de valor agregado tecnológico y actividades creativas, por un término de siete (7) años, siempre que se cumplan los siguientes requisitos:

a. Las sociedades deben tener su domicilio principal dentro del territorio colombiano, y su objeto social exclusivo debe ser el desarrollo de industrias de valor agregado tecnológico y/o actividades creativas.

b. Las sociedades deben ser constituidas e iniciar su actividad económica antes del 31 de diciembre de 2021 (Ley 2010 de 2019).

\section{Regimen Tributario Especial en Colombia.}

El Estatuto Tributario en su Artículo 19 define al Régimen Tributario Especial como:

Un conjunto de normas tributarias aplicables a un grupo de entidades, cuya finalidad es el desarrollo de su actividad meritoria y la ausencia de ánimo lucro, el tratamiento del beneficio neto o excedente se tratará como renta exenta por la destinación en dicha actividad, tendrá la aplicación de manera excepcional de una tarifa diferencial y podrá ser receptora de donaciones con un beneficio tributario directo para el donante. (ANDI, 2019)

El artículo 19 del Estatuto Tributario trata sobre los Contribuyentes del Régimen Tributario Especial, y menciona que "todas las asociaciones, fundaciones y corporaciones constituidas como ESAL, serán contribuyentes del impuesto sobre la renta $y$ complementarios, conforme a las normas aplicables a las sociedades nacionales. Excepcionalmente, podrán solicitar ante la administración tributaria, de acuerdo con el artículo 356-2, su calificación como contribuyentes del Régimen Tributario Especial, siempre y cuando cumplan con los requisitos que se enumeran a continuación:

1. Que estén legalmente constituidas.

2. Que su objeto social sea de interés general en una 0 varias de las actividades meritorias establecidas en el artículo 359 del presente Estatuto, a las cuales debe tener acceso la comunidad.

3. Que ni sus aportes sean reembolsados ni sus excedentes distribuidos, bajo ninguna modalidad, cualquiera que sea la denominación que se utilice, ni directa, ni indirectamente, ni durante su existencia, ni 
en el momento de su disolución y liquidación, de acuerdo con el artículo 356-1".

Entre las actividades meritorias se encuentran, por ejemplo, la educación, la salud, las actividades culturales, la ciencia, tecnología e innovación, las actividades de desarrollo social, actividades de protección al medio ambiente, prevención y uso de sustancias psicoactivas, promoción de actividades deportivas, actividades de desarrollo empresarial, promoción y mejoramiento de la Administración de Justicia; promoción y apoyo a otras ESAL que ejecuten acciones en el territorio nacional en este tipo de actividades meritorias; actividades de microcrédito. (Estatuto Tributario, 1989)

El régimen tributario especial, como su nombre lo indica conlleva un tratamiento diferencial para los contribuyentes que, cumpliendo las exigencias de Ley, estén sometidos a él, sí se destina, directa 0 indirectamente en el año siguiente en que se obtuvo, a programas que desarrollen su objeto social (Díaz Vargas, 2019). Así mismo la disposición en cita establece que las entidades sin ánimo de lucro cuyo objeto social principal y recursos no estén destinados a actividades de salud, educación formal, cultura, deporte aficionado, [...], o a programas de desarrollo social, o las mismas no sean de interés general, se someten al régimen ordinario del impuesto sobre la renta y complementarios (PricewaterhouseCoopers, s.f.). Es una regulación excepcional, establecida por la ley con el fin de motivar algunas actividades que por su naturaleza, fines, objetivos y forma de organización son de interés de toda la comunidad y por lo cual se ven beneficiadas con un estímulo tributario (Otálora, 2009).

El artículo 19 citado no hace referencia exclusiva a ESAL colombianas ni excluye expresamente a las ESAL extranjeras de la posibilidad de ser calificadas dentro del RTE. Ahora bien, el artículo 359 del ET, que lista las actividades meritorias, no establece que dichas actividades deban desarrollarse dentro del territorio colombiano, razón por la cual mal haríamos en darle otra interpretación a esta disposición. Por lo anterior, es posible afirmar que las ESAL extranjeras pueden pertenecer al régimen tributario especial. las ESAL extranjeras que desarrollan proyectos en varios países podrán reinvertir en otras jurisdicciones sus excedentes o beneficio neto generados en Colombia, siempre y cuando esta reinversión corresponda al desarrollo de su actividad meritoria, y no por este hecho dejarán de ser exentos (Peña, 2018).

Está establecido en las respectivas normas que podrán ser contribuyentes del Impuesto de Renta y Complementarios del Régimen Tributario Especial RTE, las asociaciones, fundaciones y corporaciones; las instituciones de educación superior aprobadas por el ICFES; los hospitales constituidos como personas jurídicas; las personas jurídicas que realicen actividades de salud que obtengan permiso de funcionamiento del Ministerio de Salud y Protección Social, y las ligas de consumidores, siempre y cuando estén constituidas como Entidades Sin Ánimo de Lucro (Nuñez Horta, 2019). Estas ESAL deben desarrollar una o más de las actividades meritorias (artículo 359 del Estatuto Tributario), deben buscar el interés general y contribuir al desarrollo integral de la sociedad o de una comunidad mediante el apoyo a sectores 0 población vulnerable, tales como: las madres cabeza de familia, la infancia, la investigación, la educación, entre otros. Así mismo, sus aportes no deben ser reembolsados, ni sus excedentes distribuidos bajo ninguna modalidad, cualquiera que sea la denominación que se utilice, ni directa ni indirectamente, ni durante su existencia, ni en el momento de su disolución o liquidación. Estas ESAL deben desarrollar una o más de las actividades meritorias (artículo 359 del estatuto tributario), deben buscar el interés general y contribuir al desarrollo integral de la sociedad o de una comunidad mediante el apoyo a sectores o población vulnerable, tales como: las madres cabeza de familia, la infancia, la investigación, la educación, entre otros. Así mismo, sus aportes no deben ser reembolsados, ni sus excedentes distribuidos bajo ninguna modalidad, cualquiera que sea la denominación que se utilice, ni directa ni indirectamente, ni durante su existencia, ni en el momento de su disolución o liquidación. (Gestionándote, 2020)

\section{RESULTADOS:}

Entidades sin Ánimo de Lucro (ESAL)

Constituidas en Bogotá D.C.

En la figura 1 se da a conocer el instrumento de investigación representado en cifras estadísticas, 
los datos son extraídos de la pagina web colombiana https://www.datos.gov.co perteneciente al MINTIC (Ministerio de Tecnologías de la Información y las Comunicaciones de Colombia), siendo propietario del conjunto de datos, la Secretaría Jurídica de la Alcadía Mayor de Bogotá D. C., la consulta de esta información se realiza el 17 de agosto del año 2020, y figura actualizada por su autor el 30 de junio de 2020.

Figura 1. Ficha técnica de la consulta.
La ficha técnica muestra la información de la entidad a cargo del resguardo de la información, que también hace referencia al lugar donde hacemos la consulta, los datos sobre la generación de datos en cuanto a idioma, fechas fecuencia y cobertura, y por último el tipo la categoría y el tipo de consulta que se hace, en este caso se consultaron las entidades sin ánimo de lucro. Castro Alfaro (2016).

Como resultado de la consulta, la plataforma nos genera un archivo en el aplicativo de Excel, el cual se analiza y permite la generación de los siguientes instrumentos estadísticos, según las entidades sin ánimo de lucro gregistradas e informadas a la Alcaldía de Bogotá (tabla 1).

\section{Información de la Entidad}

\begin{tabular}{ll}
\hline Area o dependencia & $\begin{array}{l}\text { Dirección Distrital de Inspección, Vigilancia y Control de Entidades } \\
\text { sin ánimo de lucro }\end{array}$ \\
\hline Nombre de la Entidad & Alcaldia Mayor de Bogotá \\
\hline Departamento & Bogotá D.C. \\
\hline Municipio & Bogotá D.C. \\
\hline Orden & Territorial \\
\hline Sector & Organismos de control y vigilancia \\
\hline
\end{tabular}

Información de Datos

\begin{tabular}{ll}
\hline Idioma & Español \\
\hline Cobertura Geográfica & Municipal \\
\hline Frecuencia de Actualización & Semestral \\
\hline Fecha Emisión (aaaa-mm-dd) & $2018-08-21$ \\
\hline
\end{tabular}

\section{Temas}

\begin{tabular}{ll}
\hline Categoría & Ordenamiento Territorial \\
\hline Etiquetas & entidad, sin animo de lucro \\
\hline
\end{tabular}

Fuente: Ministerio de Tecnologías de la Información y las Comunicaciones, 2020. 


\section{Tabla 1}

Entidades sin Ánimo de Lucro Inscritas en Bogotá D.C. a 30 de junio de 2020

\begin{tabular}{lr}
\hline ESAL activas & 10.455 \\
\hline ESAL liquidadas & 423 \\
\hline ESAL negadas & 20.336 \\
\hline ESAL pendientes por calificar & 4.596 \\
\hline ESAL sancionadas & 7.120 \\
\hline Otros estados & 94 \\
\hline Total esal reportadas & 43.024 \\
\hline
\end{tabular}

Fuente: Elaboración propia con base en los datos del Ministerio de Tecnologías de la Información y las Comunicaciones, 2020.

Desde que fue emitido el primer reporte el 21 de agosto de 2018 y luego de sus actualizaciones, se han reportado 43.024 Entidades Sin Ánimo de Lucro, en el Distrito capital, y a la fecha de la última actualización (30-06-2020), se evidencian 10.455 ESAL activas, las 423 liquidadas fueron las reportadas, podrían existir muchas que no funcionan pero que aún no han sido reportadas como liquidadas o en liquidación, ese gran número de 20.336 corresponde a aquellas entidades sin ánimo de lucro que se encuentran en el régimen tributario ordinario y que esperan ser calificadas como régimen tributario especial luego de que cumplan con lo estipulado en el artículo 364-5 del E.T., es importante tener claro que las ESAL pueden cambiar a régimen tributario ordinario, luego de pertenecer al régimen tributario especial, porque no actualizan de forma anual los datos como ESAL (artículo 356-3 del E.T.), o porque no tramitan la readmisión o calificación si es por primera vez que se presentan para el régimen tributario especial como ESAL.
Tabla 2

Tipos de Entidades sin Ánimo de Lucro Activas en Bogotá D.C. a 30 de junio de 2020

\begin{tabular}{lr}
\hline Asociaciones & 2.235 \\
\hline Fundaciones & 3.412 \\
\hline Sin definir por migracion & 2.281 \\
\hline Vigiladas por supersolidaria & 346 \\
\hline Otras & 2.181 \\
\hline ESAL activas & 10.455 \\
\hline Fuente: Elaboración propia con base en los datos del \\
$\begin{array}{l}\text { Ministerio de Tecnologías de la Información y las } \\
\text { Comunicaciones, 2020. }\end{array}$
\end{tabular}

Figura 2. Tipos de Entidades sin Ánimo de Lucro Activas en Bogotá D.C. a 30/06/2020.

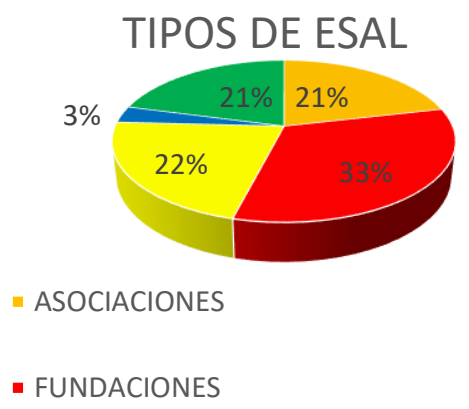

Fuente: Elaboración propia con base en los datos del Ministerio de Tecnologías de la Información y las Comunicaciones, 2020.

De las 10.455 ESAL activas en Bogotá el 33\% corresponden a Fundaciones, seguidas con un $22 \%$ que aún no han sido catalogadas, como se decía anteriormente, puede que ya sean fundaciones 0 asociaciaciones o corporaciones pero que no han actualizado la información requerida para su clasificación o que esta pendiente algún dato entre cámaras de comercio cuando se hacen traslado de domicilio de ESAL entre ciudades. Los otros grandes porcentajes (21\%), corresponden Asociaciones y otros tipos de ESAL como Clubes, Iglesias (Ej.I. Evangelicas), Academias, Instituciones, Sociedades, Corporaciones, Ligas, Comités, Colegios (ej. Colegio de Arbitros), Juntas, Congregaciones, Academias, 
Alianzas, Cabildos, Casas, Centros, círculos, comités, comunidad, Damas, Logía, Monasterios, Movimientos, Obra, Orden, Organización, Seminario y Unión.

\section{Fundación aeioTU, Ejemplo Colombiano para el Mundo}

La Fundación aeioTU es un ejemplo de transformación para la primera infancia en Colombia reconocida como fundación Carulla desde 1961, inicialmente enfocada en la educación a la población necesitada, se otorgaban becas escolares a empleados de bajos recursos de la empresa Carulla, esto durante 47 años, luego del año 2008 la fundación se proyecta como apoyo a la población mas vulnerable enfocándose en la primera infancia a través de la iniciativa aeioTU de la cual adopta su nombre actual.

Figura 3. El impacto de aeioTU en cifras.
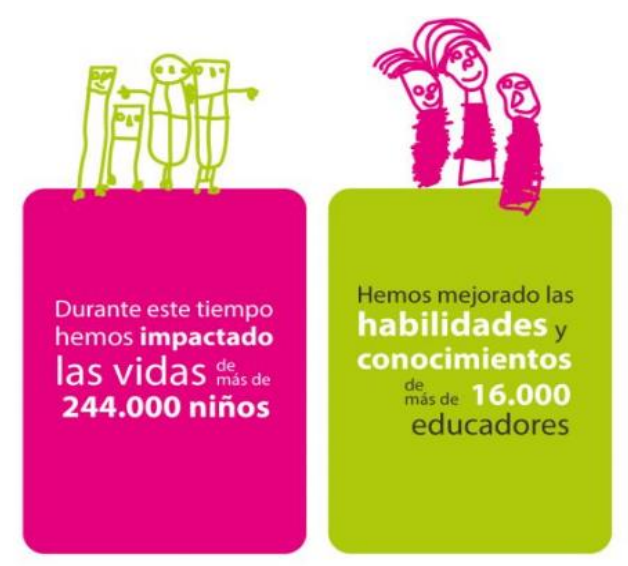

Fuente: Fundación aeioTU, s.f.a.

Esta fundación como seguramente lo hacen muchas otras, se enfoca en el desarrollo individual con proyección social, empoderando a los niños con su actuar y su sentido de pertenencia en una sociedad cambiante. En el 2017 se consolida con 30 centros educativos y una capacidad instalada para atender 14.350 niños menores de 5 años en 14 ciudades y municipios del país, cuentan con diveros miembros muy efectivos y comprometidos como por ejemplo CorpoAyapel que paso de atender 4.000 niños en el 2016 a más de 8,300 al finalizar el 2017. La fundación tambien cuenta con otros programas como el denominado desarrollo profesional para Organizaciones, es un programa para adultos donde pretende mediante talleres y metodologías guiadas, construir un mejor ambiente de calidad en las empresas, transformar las relaciones entre empleados y mejorar las operaciones al interior de las empresas (Bayona Torrente \& Bustamante, 2000).

En aeioTU se implementa una experiencia educativa innovadora, inspirada en el enfoque de Reggio Emilia, a menudo considerado como la mejor pedagogía para primera infancia en el mundo reconocida asi por la Unesco, Harvard y la Unión Europea. Es integral; y considera al niño como protagonista del aprendizaje, proceso que fortalece por medio de herramientas como el juego y el arte. Dicho enfoque es apoyado por educadores altamente capacitados con y con suficientes años de experiencia, desarrollan actividades con comunidades diversas, incluyen en su enseñanza currículos abiertos basados en el juego, aplican un programa de seguimiento al desarrollo de los niños, involucran la participación de las familias y la comunidad; promueven el uso del arte como herramienta de aprendizaje y un programa de desarrollo profesional.

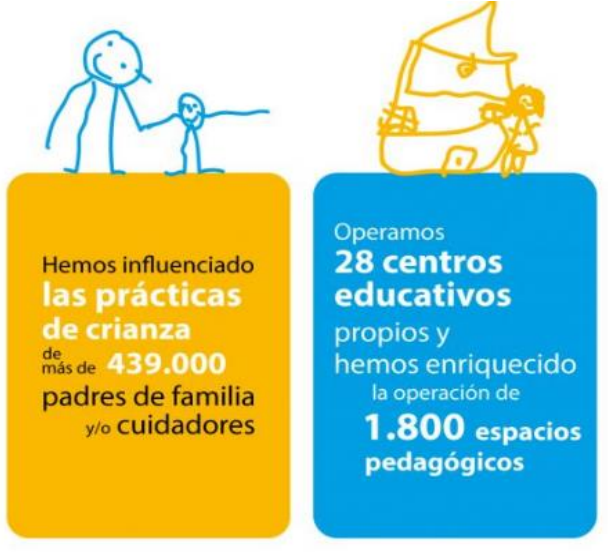


Los niños de aeioTU desarrollan su vocabulario y habilidades matemáticas y de lectura, mejoran su alimentación y se promueve su desarrollo como ciudadanos creativos, competentes y constructores de sociedad, llegan al colegio siendo líderes participativos, propositivos, argumentativos y curiosos (Fundación aeioTU, s.f.b.). Uno de los medios de sostenimiento de la Fundación es la producción de artículos como cubos de madera, chinchorros, cuentos y libros para niños, material didáctico, mandalas y juegos de mesa. Reciben donaciones en especie como ropa, juguetes que tienen como destino los niños y familias que los necesitan o son vendidos para obtener recursos destinados a salidas pedagógicas de los niños o se reinvierte en los centros de atención.

La fundación cuenta con aportes económicos de aliados públicos que cubren aproximadamente un $85 \%$ de la inversión que se requiere para mantenerla. Se reciben donaciones de particulares que apoyan el pago de salarios, mantenimiento físico, comida para los niños y compra de materiales para la dotación de recursos de aprendizaje

Figura 4. Aliados estratégicos.

Fuente: Fundación aeioTU, s.f.c.
. Otro gran apoyo que recibe la fundación es el trabajo que realizan de forma voluntaria estudiantes 0 particulares que les nace apoyar los pensamientos y filosofía de la fundación, para esto existe una preinscripción de los candidatos al voluntariado donde se evaluan los datos personales, la disponibilidad de tiempo, el área donde puede ser necesario, la zona donde se encuentra el voluntario y el objetivo por el cual se presenta como voluntario .

La fundación expuesta junto con la mayoría de ESAL cuentan con una página web donde exponen los datos de contacto para solicitar o suministrar la información necesaria, y asi poder participar de los objetivos corporativos expuestos por las fundaciones para los particulares, y también para la DIAN, la cual hace seguimiento y evalúa el desempeño de este tipo de Entidades. Uno de los fines primordiales de las fundaciones es contar con aliados estratégicos que den un soporte financiero y operativo permanente, sin ellos sería casi imposible su funcionamiento.
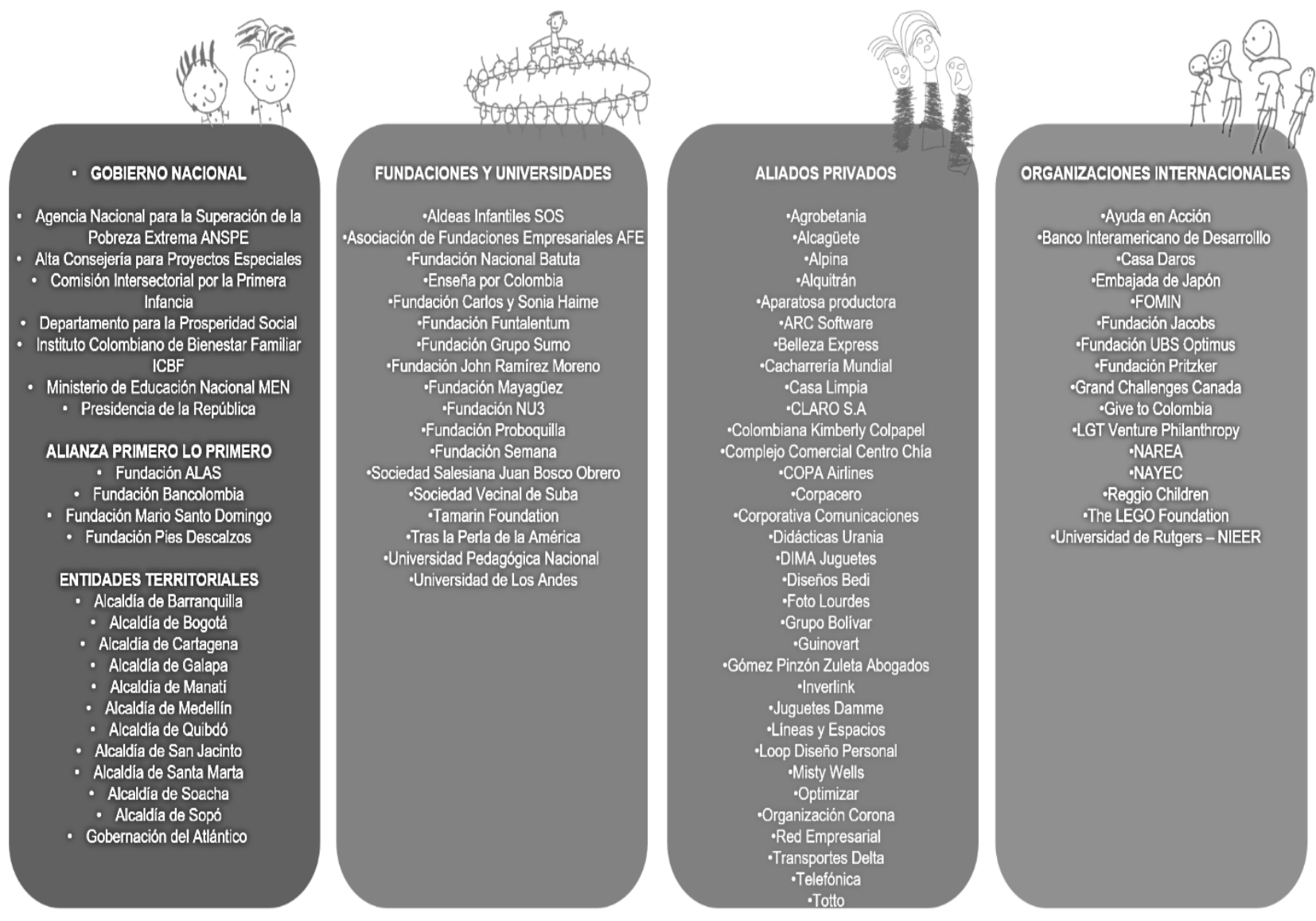
Una de las preocupaciones de los aliados estratégicos es que las fundaciones realicen el gasto de sus donaciones de forma justa, equitativa y direccionada al destino puntual, pero sobre todo que no se salga del objeto misional de la fundación, es por esto que existen medios de seguimiento ético como vínculos web hacia la página de la DIAN u otros organismos de control, 0 líneas éticas internas que vigilan y son testigos de posibles infracciones a los valores y principios organizacionales.

Por último, al igual que todas las fundaciones, aeioTU tiene un sentido amplio de publicación o divulgación, tanto de la parte financiera como de gestión; las publicaciones deben hacer ver la forma de administración y los logros obtenidos, resulta ser un medio de divulgación y de atracción a nuevos capitales, los medios de divulgación sea por página web, redes sociales 0 redes internas es muy importante hoy día para el desarrollo y progreso de estas entidades.

\section{CONCLUSIONES}

Las Entidades Sin Ánimo de Lucro (ESAL), son contituidas como personas jurídicas, que adquieren derechos y obligaciones desde su fundación; no reparten utilidades debido a su naturaleza y su objetivo social, se enfoca al beneficio social y mejoramiento de la calidad de vida del grupo hacia quien se dirigen.

Las ESAL, como personas jurídicas capaces de ejercer derechos y contraer obligaciones, así como de ser representadas judicial y extrajudicialmente, deben seguir unos lineamientos para constituirse y actuar dentro de un marco de legalidad que definitivamente garantice su adecuado desarrollo jurídico y social (Gaitán Sanchez, 2014).

Las donaciones en Colombia, dirigidas a entidades sin ánimo de lucro, resultan de gran conveniencia para Donantes y Receptores de las donaciones, actualmente el gobierno nacional apoya este tipo de movimiento financiero con la exoneración de impuestos, se requiere de la participación de personas de las empresas participantes en los procesos jurídico, estratégicos, administrativos y operacionales; las donaciones pueden ser en forma de dinero, en especie o labores.

Los incentivos tributarios, los incentivos al trabajo, o la creación de empresa en zonas especiales con tratamiento fiscal privilegiado son muy aprovechados por personas que conocen del tema, buscan el beneficio común 0 ven oportunidades de diversos negocios, desafortunadamente en Colombia aun existe mucho desconocimeinto del tema incluso por ejecutivos corporativos, quienes muchas veces no encuentran motivación personal o no divisan un lucro particular que les motive su gestión o simplemente no les interesan las tarifas tributarias ofrecidas para la reducción de impuestos.

Cada día se constituyen más y más entidades sin ánimo de lucro en Colombia, motivados por el bienestar personal de sus fundadores, o por el cumplimiento de su objetivo social, el cual radica en el apoyo general a la comunidad vulnerable, en procura de la erradicación de la pobreza. Son muchas las figuras jurídicas o tipo de empresa que existen en Colombia como entidades sin ánimo de lucro y existe un gran apoyo gubernamental, que direcciona su constitución de forma incondicional por parte de las entidades de gobierno como Cámaras de Comercio, Alcaldías, Gobernaciones o Unidades Especiales Administrativas como la DIAN.

Muchas fundaciones como aeioTU reciben donaciones no solamente en dinero sino que tambén reciben materiales para el desarrollo de actividades o el trabajo voluntario de particulares; factores que resultan importantes para el funcionamiento de estas entidades sin ánimo de lucro. También se apoyan de herramientas tecnológicas para su promosión y búsqueda de apoyos. 


\section{REFERENCIAS:}

ANDI. (2019). Guía para la Aplicación de las ESAL al Régimen Tributario Especial. ANDI. Obtenido de http://www.andi.com.co/Uploads/Gu\%C3 \%ADa\%20para\%20la\%20aplicaci\%C3\% B3n $\% 20$ de $\% 20$ las $\% 20$ ESAL $\% 20 a l \% 20 R$ TE.pdf

Arboleda, G. (2013). Proyectos: Identificación, formulación, evaluación y gerencia. Bogotá: Alfaomega.

Asesores y Consultores Internacionales, S. d. (2017). CONSULTORÍA PARA CUANTIFICAR LA EFICIENCIA DE LOS INCENTIVOS FISCALES EN EL SALVADOR. San Salvador: Asesores y Consultores Internacionales, S.A. de C.V.

Batthyány, K., \& Cabrera, M. (2011). Metodología de la investigación en ciencia sociales, Apuntes para un curso inicial. Montevideo: Universidad de la Republica.

Bayona Torrente, C., \& Bustamante, L. (2000). Las Entidades Sin Ánimo de. Bogotá: Cámara de Comercio de Bogotá.

Belalcázar Erazo, E. A., \& Ria, L. H. (2011). Derecho de las Personas Jurídicas Sin Ánimo de Lucro ONG Asociaciones, Corporaciones Fundaciones Clubes Sociales. Bogotá: Librería Ediciones del Profesional.
Beltrán, J. E. (2009). EL RÉGIMEN TRIBUTARIO ESPECIAL $Y$ SUS EFECTOS RELEVANTES EN LAS COOPERATIVAS DE TRABAJO ASOCIADO, CTA. Obtenido de file://C:/Users/USUARIO/Downloads/Dial net-

EIRegimenTributarioEspecialYSusEfecto sRelevantesEn-6634718.pdf

BID. (24 de Octubre de 2012). Banco Interamericano de Desarrollo (BID). Obtenido de Banco Interamericano de Desarrollo (BID): https://www.iadb.org/es/resourcesbusinesses/organizaciones-sin-fines-delucro-y-ambito-academico

Caicedo Silva, D. L. (2020). Análisis de los beneficios fiscales en el impuesto de renta y complementarios de las entidades sin ánimo de lucro en Colombia [Trabajo de pregrado, Universidad Cooperativa de Colombia]. Bogotá, Colombia: Repositorio Institucional.

CCB. (13 de Julio de 2020). Cámara de Comercio de Bogotá. Obtenido de Cámara de Comercio de Bogotá: http://recursos.ccb.org.co/ccb/instructivos lentidades_sin_animo_lucro/\#: :text=Defi nici $\%$ C3\%B3n\%3A\%20Entidades $\% 20$ Sin $\% 20 \%$ C3 $\% 81$ nimo $\% 20$ de,personas $\% 20$ o $\% 20$ comunidad\%20en\%20general. 
CCONG. (Agosto de 2016). Lo que hay que saber de las Entidades Sin Ánimo de Lucro. Informativo, Confederación Colombiana de ONG, Bogotá D.C., Bogotá, Colombia. Obtenido de Confederación Colombiana de

ONG: https://ccong.org.co/files/728_at_Lo\%20q ue $\% 20$ hay $\% 20$ que $\% 20$ saber $\% 20$ de $\% 201$ as\%20ESAL,\%20agosto\%20de \%202016 .pdf

CHC. (2020). Caja de Herramientas Comunitarias. Obtenido de Caja de Herramientas Comunitarias: https://ctb.ku.edu/es/tablade-contenidos/implementar/cambiandopoliticas/usar-incentivos-fiscales-paraapoyo-a-la-salud/principal

ConceptoDefinición. (26 de Julio de 2019). ConceptoDefinición. Obtenido de ConceptoDefinición: https://www.google.com.co/?gfe_rd=cr\&d cr=0\&ei=hphXWq_xJI_Q8AesslqICA

Dávila, J. C. (2012). La doble dimensión de una capacidad organizacional evidencias de una organización sin ánimo de lucro que compite en el mercado. Cuadernos de Administración, 11-37.

DIAN. (27 de Abril de 2018). Concepto No. 0481 ESAL y Donaciones. 27 de Abril de 2018. Obtenido de Departamento de Impuestos y Aduanas Nacionales: https://www.dian.gov.co/normatividad/Do cuments/Concepto_unificado_ESAL_No_ 481_27042018.pdf
Díaz Vargas, L. L. (2019). Aplicación de las disposiciones tributarias actuales en las entidades sin ánimo de lucro en Colombia respecto al impuesto de renta y complementarios (Tesis de pregrado). . Universidad Cooperativa de Colombia.

E.T. (13 de Julio de 2020). Estatuto Tributario. Obtenido de Estatuto Tributario: https://estatuto.co/?e=1326

Economía, A. e. (12 de Octubre de 2010). Auditoría en Contabilidad \& Economía. Obtenido de Auditoría en Contabilidad \& Economía: https://blogconsultorasur.wordpress.com/ 2010/10/12/donaciones/

Flórez Gómez, A. M. (2018). Acompañamiento contable y tributario a las asociaciones de mujeres "COISDES" de la ciudad de Ibagué (Tesis de pregado). Obtenido de http:/hdl.handle.net/20.500.12494/13806

Fortún, M. (2020). ECONOMIPEDIA. Obtenido de ECONOMIPEDIA: https://economipedia.com/definiciones/in centivo-fiscal.html

Fundación aeioTU. (18 de 08 de 2020). https://www.aeiotu.com. Obtenido de https://www.aeiotu.com/home/2391-2/laexperiencia-educativa-aeiotu/

Gaitán Sanchez, O. (2014). Guía Práctica de las Entidades sin Ánimo de Lucro y del Sector Solidario. Bogotá: Kimpres. 
Juridica., E. (05 de 2021). Enciclopedia Juridica. Obtenido de http://www.enciclopediajuridica.com/d/donatario/donatario.htm impuesto sobre la renta. Obtenido de Gerence.com:

https://www.gerencie.com/estructura-delimpuesto-sobre-la-

renta.htm|\#: :text=El\%20r\%C3\%A9gime $\mathrm{n} \% 20$ ordinario\%20en\%20el,del\%20impu esto $\% 20 a \% 201 a \% 20$ renta.

Gestionándote. (Marzo de 2020). Gestionándote, Subvenciones y Recursos para la Gestión Social. Obtenido de Las ESAL y el Régimen Tributario Especial en Colombia - Parte 1: https://gestionandote.org/lasesal-y-el-regimen-tributario-especial-encolombia-parte-1/

González Robles, M. (2019). Responsabilidades de los Administradores en las Entidades sin ánimo de lucro-ESAL. Revista Civilizar Ciencias Sociales y Humanas, 19-37.

Hernández Sampieri, R., Fernández Collado, C., \& Baptista Lucio, M. (2010). Metodología de la Investigación. México: McGraw-Hill.

Jaramillo Díaz, J. (2010). Entidades sin ánimo de lucro caracteristicas y aplicaciones del régimen tributario. Bogotá: Legis S.A.

Jaramillo Díaz, J., Vargas Ballén, J., \& Durán Acosta, F. (1999). Entidades sin Ánimo de Lucro Régimen Tributario Especial. Bogotá: Legis.

JURÍDICA, E. (2020). ENCICLOPEDIA JURÍDICA. Obtenido de ENCICLOPEDIA JURÍDICA: http://www.enciclopediajuridica.com/d/donaci\%C3\%B3n/donaci\% C3\%B3n.htm
Kaldor, M. (2003). Global Civil Society: An Answer to War. Cambridge: Polity Press.

Kerlinger, F. (1997). Investigación del Comportamiento. México: Mc Graw Hill.

KLUWER, W. (2020). WOLTERS KLUWER. Obtenido de WOLTERS KLUWER: https://guiasjuridicas.wolterskluwer.es/Co ntent/Documento.aspx?params=H4sIAA AAAAAEAMtMSbF1jTAAAUMjE0tLtbLUo uLM_DxblwMDCwNzAwuQQGZapUtckhlQaptWmJOcSoAwl_KtjUAAAA=WKE

Martínez Ravé, G. M. (2003). Responsabilidad civil extracontractual. Bogotá: Temis.

Minjusticia. (27 de Diciembre de 2019). Sistema Único de Información Normativa. Obtenido de Sistema único de Información Normativa: http://www.suinjuriscol.gov.co/viewDocument.asp?ruta= Leyes/30038705

N.U. (2018). Diseño y Evaluación de Incentivos Tributarios en Países en Desarrollo. Derechos de Naciones Unidas New York 2018.

Niño Mejia, J. (2017). La tributación de las entidades sin ánimo de lucro y el régimen tributario especial en Colombia. Revista Instituto Colombiano de Derecho Tributario, 27-65.

Niño Mejía, J. A. (2007). La tributación de las entidades sin ánimo de lucro y el régimen tributario especial en Colombia. Revista Instituto Colombiano de Derecho Tributario, 27-66. 
Nuñez Horta, C. y. (2019). Estructura financiera de las entidades sin ánimo de lucro. Obtenido de http://hdl.handle.net/20.500.12494/13394

Parada, J. R. (2001). Aspectos Económicos de empresas sin fines de lucro, caridad y donación. Revista Universidad EAFIT, 5565.

Peña, J. A. (Jueves 29 de Noviembre de 2018). Régimen tributario especial para Esal extranjeras. Obtenido de Asuntos: Legales:

https://www.asuntoslegales.com.co/anali sis/julian-andres-pena-2525661/regimentributario-especial-para-esal-extranjeras2799332

Price Waterhouse Coopers Servicios Legales y Tributarios Ltda, P. (s.f.). R.T.E. Concepto I ESAL Requisitos para acceder al régimen tributario especial. Obtenido de R.T.E. Concepto / ESAL Requisitos para acceder al régimen tributario especial.: https://www.pwcimpuestosenlinea.co/Re positorio\%20PwC/PDF/Jurisprudencia/C onsejo\%20de\%20Estado/Secci\%C3\%B3 n\%204/Sentencias/2011/SEN-Exp016285-11-D.pdf

Reyes Villamizar, F. (2006). Derecho Societario. Bogotá: Temis S.A.

Rosenau, J. (1997). Cambio y complejidad: Desafíos para la comprensión en el campo de las relaciones internacionales. Análisis Político, 105-120.

Ruiz de Olabuenaga, J. (2005). El tercer sector español y sus campos de actuación. Revista Española del Tercer Sector, 112123.
Salamon, L. (1999). La sociedad civil global: Las dimensiones del sector no lucrativo. Bilbao: Fundación BBVA.

Sánchez, Ó. M. (2014). Guía Práctica de las Entidades sin Ánimo de Lucro y del Sector Solidario. Bogotá, D.C., Colombia.: Kimpres LDTA.

Sorj, B. (2007). ¿Pueden las ONG reemplazar al Estado?: Sociedad civil y Estado en América Latina. Nueva Sociedad, 126140.

Tamayo y Tamayo, M. (2004). Diccionario de la Investigacion Científica. México: Limusa.

Vargas, H. J. (1992). Acerca de la naturalezay evolución de los organismos no gubernamentales (ONG) en Colombia: Bases para la compresión del fenómeno. Bogotá: Fundación Social.

Villar, R. (2001). El tercer sector en Colombia: Evolución, dimensión y tendencias. Bogotá: CCONG/Fundación DIS.

VTF. (2020). VTF, Vocabulartio en Términos Fiscales. Obtenido de VTF, Vocabulartio en Términos Fiscales: http://www.ub.edu/gidei/vocabulari/es/no de/808\#: :text=Est\%C3\%ADmulo\%20en $\% 20$ forma $\% 20$ de $\% 20$ bonificaciones,por $\% 20$ parte $\% 20$ del $\% 20$ sector $\% 20$ p $\%$ C3\% BAblico. 